\title{
Antipollution skin protection - a new paradigm and its demonstration on two active compounds
}

This article was published in the following Dove Press journal:

Clinical, Cosmetic and Investigational Dermatology

17 May 2017

Number of times this article has been viewed

\author{
Meital Portugal-Cohen ${ }^{1,2}$ \\ Miriam Oron ${ }^{1,2}$ \\ Dror Cohen ${ }^{1-3}$ \\ Zeevi Ma'or ${ }^{1,2}$
}

'AHAVA Dead Sea Laboratories, Lod, Israel; ${ }^{2}$ The Dead Sea Laboratory for Skin Biochemistry and Biotechnology, Dead Sea and Arava Science Center, Masada, Israel; ${ }^{3}$ The Institute for Drug Research, The Hebrew University of Jerusalem, Jerusalem, Israel
Correspondence: Meital Portugal-Cohen AHAVA Dead Sea Laboratories, ARAVA I Street, PO Box 109, Lod 70150, Israel Tel +972523407625

Fax +972 3557 IIII

Email meital.p@ahava.com
Background: Urban pollution is a major source of concern for human health and is a complex of many environmental factors. The topical exposure to pollution activates cutaneous stress.

Objective: In this study, we tested the antipollution protection of two active components: Dead Sea minerals (Dead Sea mineral-rich water [DSW]) and anionic polysaccharide (PolluStop ${ }^{\circledR}$ [PS]).

Materials and methods: Two representative pollution models were studied using reconstructed epidermis: 1) mixture of pollutants (MOP) containing heavy metals and atmospheric particulate matter and 2) ozone exposure. DSW and PS were topically applied alone or in combination, and their protection against pollution was assessed by testing the levels of the inflammation markers interleukin $1 \alpha(\mathrm{IL}-1 \alpha)$ and prostaglandin E2 (PGE2).

Results: MOP exposure induced IL-1 $\alpha$ release, which was attenuated following pre-application with DSW and PS alone or in combination. Ozone exposure induced IL-1 $\alpha$ and PGE2 release. Pre-application with DSW or PS alone did not inhibit IL- $1 \alpha$ and PGE2 overproduction. Only when DSW and PS were mixed together, inhibition of these inflammatory markers was observed. Conclusion: The observations reveal the potential use of active agents in combination for a selective mode of protection from urban pollution. This is because many active materials cannot solely provide a broad protection against different types of pollutants. This strategy might be beneficial for future antipollution regimen formulated in both pharmaceutical and cosmetic products.

Keywords: urban pollution, dermal exposure, inflammation, oxidation, alternative skin models, antipollution activity

\section{Introduction}

The vast urbanization of last decades had amplified health risks associated with skin exposure to pollution, making the pollution one of the most important sources of concern for modern human health. ${ }^{1,2}$ While traditional pollution was classified as associated with smoke and smog, nowadays, pollution has a more complex and dynamic definition due to accumulating data on its different factors and triggers, their mode of action and the variation in exposure extent.

The World Health Organization (WHO) defines pollution as contamination of the indoor or outdoor environment by any chemical, physical or biological agent that modifies the natural characteristics of the atmosphere. ${ }^{2}$ Air pollution caused by industry, vehicle fumes, cigarette smoke and other sources together with solar radiation, especially the ultra violet (UV) ranges, is known to have a detrimental effect on human skin and body health altogether and leads to various serious pathologies. 
Pollution can be classified according to its causes into primary pollutants and secondary pollutants. ${ }^{1}$ Primary pollutants comprises two main groups: particulate matter (PM) and toxic gases, basically compounds of $\mathrm{CO}_{2}, \mathrm{CO}, \mathrm{SO}_{2}, \mathrm{NO}$, $\mathrm{NO}_{2}$ and other nitrogen oxides $\left(\mathrm{NO}_{x}\right)$, such as $\mathrm{N}_{2} \mathrm{O}, \mathrm{N}_{2} \mathrm{O}_{2}$, $\mathrm{N}_{2} \mathrm{O}_{3}, \mathrm{~N}_{2} \mathrm{O}_{4}$ and $\mathrm{N}_{2} \mathrm{O}_{5}$, including small molecular weight hydrocarbons, e.g., volatile organic compounds (VOC). The pollutants caused by PM may also carry polyaromatic hydrocarbons (PAHs), which are highly lipophilic and therefore easily penetrate through the skin barrier. ${ }^{3}$ Polychlorinated biphenyls (PCBs) are another toxic PM. They are widespread persistent organic pollutants in the atmosphere, and because of their persistence, toxicity, bioaccumulation and longdistance migration, they can also be passed on to offspring through the cellular matrix, causing deformities. ${ }^{4}$

PM plays a major role in primary pollution. It comprises toxic organic compounds, heavy metals, driving automobile smoke and burning plants and smoke, dirt and dust from industrial factories, agricultural farming and roads.

Under certain atmospheric conditions, secondary pollutants, such as ozone and peroxyacetyl nitrates (PANs), are formed by photochemical reactions of the primary pollutants exposed to heat and UV radiation. These newly formed pollutants accumulate in the low atmospheric level, known as the troposphere, and settle over both urban and rural areas, forming what is usually known as smog. ${ }^{1}$

The level of pollution and pollutant composition vary throughout the time of the day, differ by season and geographic location and are affected by local environmental human activity. In particular, ozone levels increase during summer, when the strong sunshine enhances ozone production via photochemical reactions of the primary pollutants. Nitrous gases, mainly from automobile combustion and industry, are generally higher in South American and Far East Asian big cities and some European cities due to their high industrial activities. In general, we may state that the kind of pollution we are exposed to is mainly defined by time and place factors.

The topical exposure to pollution may activate cutaneous stress since pollutants can react with skin tissue, alter functioning of the skin barrier, penetrate the skin barrier and cause oxidative stress and inflammation damages by reacting with skin proteins, lipids and DNA molecules. ${ }^{5}$

The damaging effects of skin exposure to pollutants may result in skin disorders and pathologies, including xerotic skin, sensitive skin, premature skin aging and accelerated aging symptoms, such as wrinkle formation, abnormal pigmentation and skin dryness. ${ }^{6-9}$ Pollutants may also be involved in acne, eczema, skin rashes and skin cancers. ${ }^{10,11}$ Therefore, effective protection of skin against health risks related to its exposure to pollution is very important not only for beauty reasons or for attenuating cutaneous manifestation but also for the inhibition of health risks involving intrinsic damage to other organs.

Prolonged and repetitive daily exposure to high levels of pollutants impairs the skin's natural defense capacity to some extent. Moreover, some pollutants (e.g., ozone) can induce damage via signal transduction mechanism even when there is no percutaneous penetration to deeper skin layers. ${ }^{12}$ Therefore, we cannot rely on skin's natural resilience for daily skin protection. There is a real need for an effective shield against these modern detrimental consequences of different "cocktails" of polluting agents. Daily application of skincare products, formulated with active ingredients, known to improve skin barrier function, do so by physically shielding the skin. Some ingredients work by scavenging the oxidative pollutants. Film formers and antioxidants do not always provide the sufficient protection. Indeed, they can reduce immediately the exposure of skin to the pollution to some extent and its consequential risk of health damage in the short term; however, this regimen is not expected to be effective in eliminating the long-term chronic exposure risks. There is a need for a novel antipollution skin-protecting strategy, considering the different risks, and supplying a multipurpose treatment that combines biological and physical defense against contemporary pollution risks in both shortand long-term exposure.

In this study, we demonstrate a new paradigm of treatment against pollution based on two active components, which can be potent for modern antipollution defense.

Dead Sea minerals are well known for their therapeutic efficacy in treating a variety of skin conditions as well as for their cosmetic benefits. ${ }^{13-16}$ Dead Sea mineral-rich water (DSW; Osmoter ${ }^{\mathrm{TM}}$, a natural commercial composition of Dead Sea water) was shown to reduce skin senescence markers, to attenuate the expression of UVB damage biomarkers ${ }^{17}$ and to improve skin smoothness. ${ }^{14}$ Nevertheless, the topical application of Dead Sea minerals for skin protection against pollution damage has never been tested.

PolluStop ${ }^{\circledR}$ (PS) is a patented commercial cosmetic active ingredient, which comprises an anionic polysaccharide of a high molecular weight. The polysaccharide is obtained by a controlled biotechnological process, and when formulated in a cosmetic preparation and topically applied, it protects human skin from pollution damage. This anionic polysaccharide shields the skin by matrix forming 
and is proven to serve as a physical barrier to the following three types of pollution stresses: atmospheric pollution (carbon particles, PM and heavy metals), domestic pollution (chemicals) and UV radiation. As such, PS limits the extracellular and intercellular damages induced by such pollution stresses, e.g., oxidative stress, inflammation and mitochondrial toxicity. ${ }^{18}$

The capabilities of DSW and PS, as stand-alone and in combination, to protect human skin against primary and secondary pollution types were investigated using in vitro human skin models based on reconstructed epidermis. The results indicate selective skin protection against a broad range of pollutants by the tested ingredients. A new versatile strategy for skin protection from modern pollutant health risks, which was lately submitted as a patent pending (IL patent no 247192), is presented in this work, based on a mixture of the two cosmetic active ingredients.

\section{Materials and methods \\ Preparation of test materials}

Test materials consist of either Dead Sea water extract (Osmoter), either biosaccharide gum-4 or 1,2-hexanediol (PS) or their combination.

Osmoter (DSW): the major constituents of Osmoter are the following ions: $\mathrm{Mg}_{2}+(92,500 \mathrm{mg} / \mathrm{L}), \mathrm{Ca}_{2}+(38,000 \mathrm{mg} / \mathrm{L})$, $\mathrm{K}+(1400 \mathrm{mg} / \mathrm{L}), \mathrm{Na}+(2000 \mathrm{mg} / \mathrm{mL}), \mathrm{Sr}_{2}+(800 \mathrm{mg} / \mathrm{L}), \mathrm{Cl}-$ $(345,000 \mathrm{mg} / \mathrm{L})$ and $\mathrm{Br}-(11,500 \mathrm{mg} / \mathrm{L})$.

PS was obtained as a commercial active ingredient from Solabia Group, Pantin Cedex, France.

Osmoter and PS were tested in different ratios, as described in Table 1.

\section{Skin model}

The preparations in Table 1 were tested on reconstructed epidermis. These are normal human-derived epidermal keratinocytes that have been cultured to form a threedimensional multilayered, highly differentiated model of the human epidermis. The model exhibits normal barrier

Table I Preparation of test materials in different concentrations

\begin{tabular}{ll}
\hline DSW & PS \\
\hline- & $5 \%(w / w)$ \\
$0.25 \%(w / w)$ & - \\
$0.5 \%(w / w)$ & - \\
$0.8 \%(w / w)$ & - \\
$0.25 \%(w / w)$ & $5 \%(w / w)$ \\
$0.5 \%(w / w)$ & $5 \%(w / w)$ \\
$0.8 \%(w / w)$ & $5 \%(w / w)$ \\
\hline
\end{tabular}

Abbreviations: DSW, Dead Sea mineral-rich water; PS, PolluStop. functions (presence of a well-differentiated stratum corneum). The skin models were supplied by MatTek EpiDerm for the ozone pollution model and by CellSystem (Troisdorf, Batch 100-AF0828-1) for the PM and heavy metal pollution models.

\section{Tissue preparation}

Upon arrival, the epidermis tissues were stored at $4{ }^{\circ} \mathrm{C}$ until used. Prior to use, the tissues were removed from the agarose-shipping tray and placed into a six-well plate containing $0.9 \mathrm{~mL}$ of hydrocortisone free assay medium $\left(37^{\circ} \mathrm{C}\right)$. The tissues were allowed to incubate for at least 1 hour at $37^{\circ} \mathrm{C}$ and $5 \% \mathrm{CO}_{2}$. After this initial incubation, the assay medium was replaced with $0.9 \mathrm{~mL}$ of fresh medium $\left(37^{\circ} \mathrm{C}\right)$ and the tissues were treated overnight topically with either the test materials, $100 \mathrm{mg} / \mathrm{mL}$ Trolox or left untreated. Four tissue samples were prepared for each treatment.

\section{Primary pollution model - mixture of heavy metal and atmospheric PM}

A reconstructed human skin model was used comprising normal human epidermal keratinocytes, growing as an integrated three-dimensional cell culture model, perfectly mimicking the human skin in vitro. The model exhibits normal barrier functions (presence of a well-differentiated stratum corneum). It was supplied by CellSystem (Troisdorf, Batch 100-AF0828-1).

\section{Treatment and exposure}

Epidermis tissues were exposed to a mixture of pollutants (MOP) composed of heavy metal and atmospheric PM. The composition of heavy metals is described in Table 2.

Atmospheric PM was obtained as a standard composition consisting of 23 polyhalogenated aromatic compounds (PHAs), 13 PCBs and four chlorinated pesticides. This composition was approved by the National Institute of Standards and Technology (NIST), USA, certificate 1649b.

Table 2 Heavy metals composition

\begin{tabular}{ll}
\hline Element & Mass fraction $(\mathbf{p p m})$ \\
\hline As & $115.5 \pm 3.9$ \\
$\mathrm{Cd}$ & $73.7 \pm 2.3$ \\
$\mathrm{Co}$ & $17.93 \pm 0.68$ \\
$\mathrm{Cr}$ & $402 \pm 13$ \\
$\mathrm{Ni}$ & $81.1 \pm 6.8$ \\
$\mathrm{~Pb}$ & $0.655 \pm 0.033$ \\
$\mathrm{Sr}$ & $215 \pm 17$ \\
$\mathrm{Sb}$ & $45.4 \pm 1.4$ \\
\hline
\end{tabular}




\section{Epidermis exposure to MOP and treatment with test materials}

A total of $30 \mathrm{~mL}$ of test materials sample (DSW, PS or their combination) was applied on the epidermis together with $100 \mu \mathrm{L}$ of MOP. The epidermis units were daily washed with phosphate-buffered saline (PBS), and the samples were applied again. Exposure to MOP was carried out at $37^{\circ} \mathrm{C}$ and $5 \% \mathrm{CO}_{2}$. A total of $100 \mu \mathrm{L}$ of PBS served as negative control (NC). After 48-hour incubation, changes in tissue viability were determined using a 3-(4,5-dimethylthiazol2-yl)-2,5-diphenyltetrazolium bromide (MTT) assay, while the cell culture medium was collected and stored at $-75^{\circ} \mathrm{C}$ until analyzed for evaluation of IL-1 $\alpha$ levels. All the experiments were carried out in three replicates.

\section{Epidermis exposure to ozone and treatment with test materials}

Following the overnight incubation, the tissues were rinsed with PBS to remove any test materials and placed into a 24-well plate containing $300 \mu \mathrm{L}$ of PBS per well. An inflammatory response in the tissues was then initiated via ozone exposure. For the exposure, the tissues were placed into an exposure chamber filled with ozone ( $20 \mathrm{ppm})$ for 1 hour. One set of tissues was not exposed to ozone and served as a non-ozone exposed control. After the ozone exposure, the test materials were reapplied to the tissues and the tissues were incubated overnight in fresh media. After the 24-hour incubation, changes in tissue viability were determined using an MTT assay, while the cell culture medium was collected and stored at $-75^{\circ} \mathrm{C}$ until analyzed for interleukin $1 \alpha(\mathrm{IL}-1 \alpha)$ and prostaglandin $\mathrm{E}_{2}$ (PGE2).

\section{MTT cell viability assay}

Epidermis units were treated with $1 \mathrm{mg} / \mathrm{mL}$ MTT solution for 3 hours at $37^{\circ} \mathrm{C}$. The solution was then removed and replaced with isopropanol, with further 2 hours incubation at room temperature. Two aliquots of every sample were transferred to a 96-well plate for the reading. The absorbance was read at the wavelength of $540 / 570 \mathrm{~nm}$ with a colorimeter (Tecan, model Sunrise remote) equipped with a microplate reader.

\section{IL-I $\alpha$ measurement}

IL- $1 \alpha$ had been determined in the culture media of treated and untreated epidermis using a direct enzyme-linked immunosorbent assay (ELISA) test. A total of $100 \mu \mathrm{L}$ of the samples were incubated in a plate pre-coated with a primary anti-cytokine antibody for 2 hours at room temperature. At the end, the samples were washed with a suitable buffer. Then,
$100 \mu \mathrm{L}$ of secondary biotinylated antibody was added with a further 2-hour incubation step at room temperature. The samples were washed with a suitable buffer and incubated for 30 minutes with streptavidin peroxidase solution. After washing for five times, a colorimetric substrate (hydrogen peroxide + tetramethylbenzidine as a chromagen) was added to each well. Once a sufficient level of color development had occurred, $50 \mu \mathrm{L}$ of stop solution ( $2 \mathrm{~N}$ sulfuric acid) was added to each well and the plate was read at $460 \mathrm{~nm}$.

\section{Measurement of PGE2 secretion}

The culture media of treated skin pieces were collected 24 hours following treatment, and the secreted PGE2 levels were measured using ELISA assay.

A series of PGE2 standards were prepared ranging from 7.8 to $1000 \mathrm{pg} / \mathrm{mL}$. An ELISA plate was prepared by removing any unneeded strips from the plate frame, remembering to designate two wells each for total activity (TA) wells, nonspecific binding (NSB) wells, maximum binding (MB) wells and substrate blank wells (B0). A total of $150 \mu \mathrm{L}$ of the tissue culture medium was added to the NSB wells, while $100 \mu \mathrm{L}$ of the medium was added to the B0 wells. A total of $100 \mu \mathrm{L}$ of standard or sample was then added to respective wells. To each of the wells used (except the TA and B0), $50 \mu \mathrm{L}$ of the PGE2 alkaline phosphatase conjugate was added. Next, $50 \mu \mathrm{L}$ of PGE2 alkaline phosphatase antibody solution was added to each well (except the TA, NSB and SD wells). The plates were covered and incubated at $2^{\circ} \mathrm{C}-8^{\circ} \mathrm{C}$ for $18-24$ hours. After the incubation, each well was washed three times with $400 \mu \mathrm{L}$ of wash buffer. After the last wash, $5 \mu \mathrm{L}$ of PGE2 alkaline phosphatase conjugate was added to the TA wells and $200 \mu \mathrm{L}$ of fresh Ellman's reagent was added to each well. The plate was incubated at room temperature with periodic readings of the absorbance $(405 \mathrm{~nm})$ using a plate reader.

\section{Data analysis}

Values are expressed as mean \pm standard deviation. Differences between average values were tested for significance using the Student's $t$-test and considered significant for $p \leq 0.05$.

\section{Results}

In this study, two skincare commercial materials, 1) DSW - a Dead Sea water extract and 2) PS - a polysaccharide - biosaccharide gum-4 or 1,2-hexanediol, were tested for their antipollution defense in human-reconstructed skin. Two representative pollution models were conducted: 1) MOP containing heavy metals as described in Table 2, at atmospheric 
PM, for primary pollution and 2) ozone exposure, for secondary pollution.

Treatment with DSW $(0.25 \%, 0.5 \%, 0.8 \%)$ and PS (5\%) alone or in combination in unstressed (normal) reconstructed epidermis tissues did not affect their viability (data are not shown). Thus, DSW and PS were further tested using the two in vitro pollution models.

Pollution damage was assessed by the inflammatory biomarkers IL- $1 \alpha$ and PGE2 additionally to epidermal viability.

IL- $1 \alpha$ is an epidermal cytokine that is constitutively produced in the keratinocytes in substantial amounts and plays an important role in normal skin homeostasis. ${ }^{19}$ It is a regulator of skin architecture and functions (e.g., collagen, synthesis, melanogenesis, skin barrier function). Although it is essential for normal skin functioning, overproduction of this cytokine, even in small amounts, might lead to adverse effects. IL- $1 \alpha$ has been identified as a master mediator of skin irritation and inflammation. Prostaglandins are synthesized in a variety of cells from arachidonic acid. Arachidonic acid can be released from membrane phospholipids via phospholipase A2 and is then committed to form PGEs via the action of cyclooxygenase. PGE2 has been shown to be involved in the inflammatory pathway of the skin..$^{20,21}$

\section{Pollutant mixture results}

MOP exposure of the epidermis equivalents resulted in a significant increase in the release of IL- $1 \alpha$ by 44 fold, along with a significant sharp decrease in tissue viability by $74 \%$ (Figures 1 and 2, respectively). These observations indicate

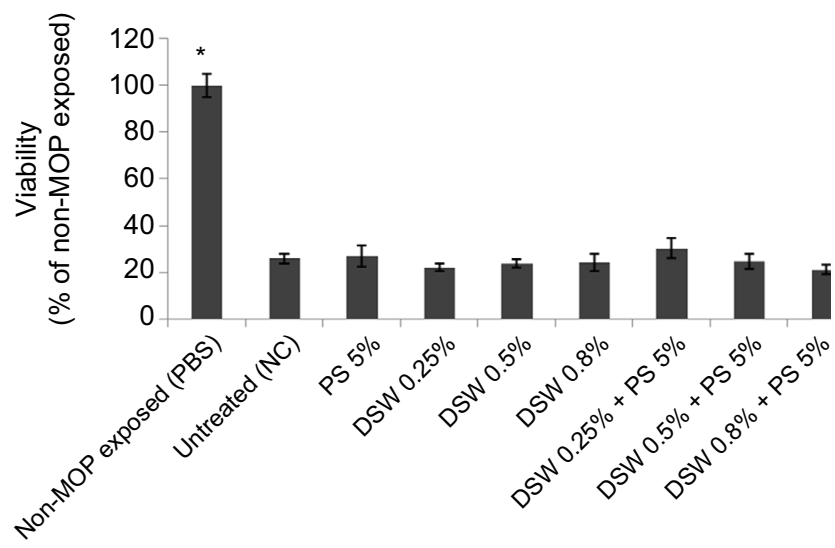

Figure I Epidermal viability following an MOP exposure to DSW and PS treatments. Notes: A total of $30 \mu \mathrm{L}$ of the sample has been applied on the epidermis with $100 \mu \mathrm{L}$ of MOP or PBS (non-MOP exposed) and maintained for 48 hours on the epidermis. Every day, the epidermis units were washed with PBS and the samples were applied again. At the end of the exposure period (48 hours), the MTT assay was performed to evaluate the cell survival. Values are expressed as mean \pm standard deviation. *Values that are significantly different from the untreated tissues $(p<0.05)$.

Abbreviations: MOP, mixture of pollutants; DSW, Dead Sea mineral-rich water; PS, PolluStop; PBS, phosphate-buffered saline; MTT, 3-(4,5-dimethylthiazol-2-yl)-2,5diphenyltetrazolium bromide; NC, negative control.

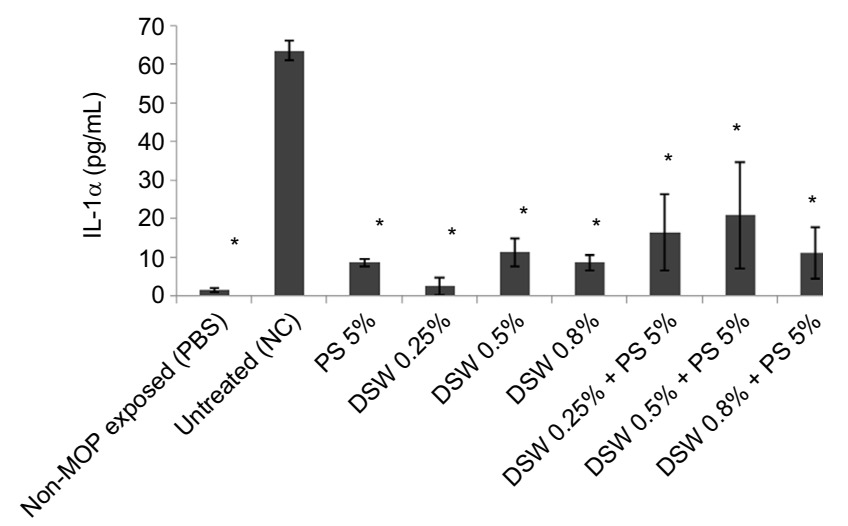

Figure $2 \mathrm{IL}-\mathrm{I} \alpha$ levels following MOP exposure to DSW and PS treatments.

Notes: A total of $30 \mu \mathrm{L}$ of the sample has been applied on the epidermis with $100 \mu \mathrm{L}$ of MOP or PBS (non-MOP exposed) and maintained for 48 hours on the epidermis. Every day, the epidermis units were washed with PBS and the samples were applied again. At the end of the exposure period (48 hours), the culture medium was collected for IL-I $\alpha$ determination using ELISA assay. Values are expressed as mean \pm standard deviation. *Values that are significantly different from the untreated tissues $(p<0.05)$.

Abbreviations: IL-I $\alpha$, interleukin I $\alpha$; MOP, mixture of pollutants; DSW, Dead Sea mineral-rich water; PS, PolluStop; PBS, phosphate-buffered saline; ELISA, enzymelinked immunosorbent assay; NC, negative control.

the inflammation-related damage to epidermis following exposure to PM and heavy metals.

Treatments with all test materials, i.e., DSW, PS and their combinations, in different ratios demonstrated a significant inhibition in IL- $1 \alpha$ release at a range of $37 \%-65 \%$. DSW $0.25 \%$ alone showed the highest IL- $1 \alpha$ inhibition (65\%), similar to the samples tested in combination with PS 5\% (63\%; Figure 2).

None of the test materials and their combination fully inhibited the sharp decrease in epidermal viability following MOP exposure nor did they contribute to its decrease following MOP exposure. Hence, for MOP exposure, DSW and PS provide significant protection only against IL-1 $\alpha$ overproduction.

\section{Ozone exposure results}

Ozone exposure of the epidermis equivalents resulted in a significant increase in the release of both IL- $1 \alpha(320 \%)$ and PGE2 (55\%), along with a mild decrease in tissue viability (17\%; Figures 3-5, respectively). The increase in IL-1 $\alpha$ secretion and the decrease in cell viability following MOP exposure were much higher than the decrease in cell viability following ozone exposure.

Neither DSW nor PS was observed to reduce the release of either one or both inflammatory markers induced by ozone exposure (IL-1 $\alpha$ and PGE2). Only when DSW and PS were mixed together, a significant inhibition in one or both inflammatory markers was observed. Treatment with a mixture containing DSW $0.8 \%$ + PS 5\% significantly reduced 


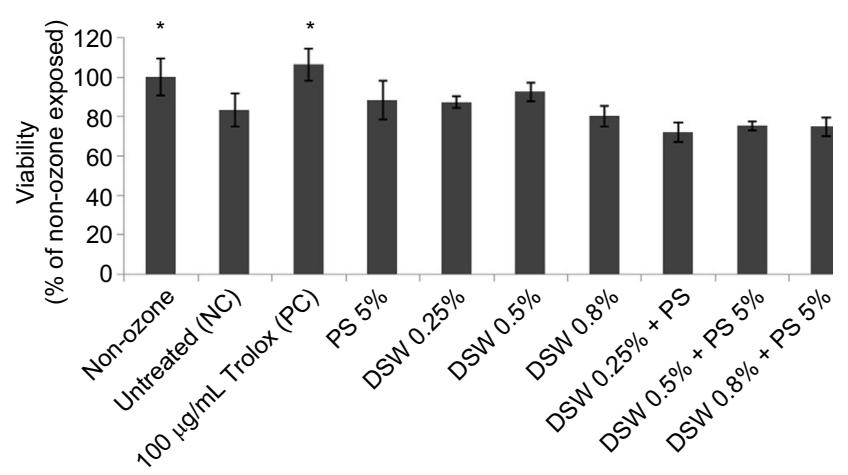

Figure 3 Epidermal viability following ozone exposure to DSW and PS treatments. Notes: Epidermis equivalents were treated topically with test materials. Following overnight incubation, they were exposed to $20 \mathrm{ppm}$ ozone for I hour and test materials were reapplied. After 24 hours incubation, tissue viability was tested using the MTT assay. Viability is expressed as a percentage of the non-ozone exposed (non-inflamed) controls, which are used to represent $100 \%$ viability. Values are expressed as mean \pm standard deviation. *Values that are significantly different from the untreated tissues $(p<0.05)$.

Abbreviations: DSW, Dead Sea mineral-rich water; PS, PolluStop; MTT, 3-(4,5-dimethylthiazol-2-yl)-2,5-diphenyltetrazolium bromide; NC, negative control; PC, positive control.

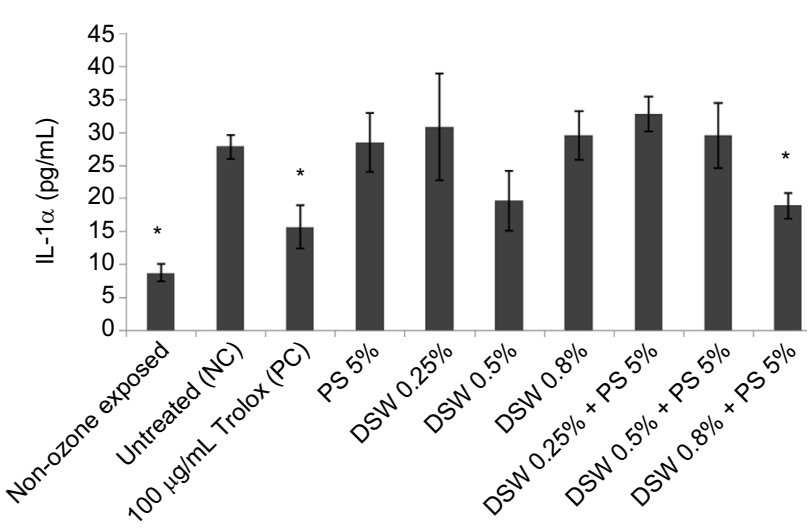

Figure 4 IL-I $\alpha$ levels following ozone exposure to DSW and PS treatments. Notes: Epidermis equivalents were treated topically with test materials. Following overnight incubation, they were exposed to $20 \mathrm{ppm}$ ozone for I hour and test materials were reapplied. After 24 hours of incubation, the medium was collected for IL-I $\alpha$ analysis. IL-I $\alpha$ values are expressed as concentrations. Values are expressed as mean \pm standard deviation. *Values that are significantly different from the untreated tissues $(p<0.05)$.

Abbreviations: IL- $\mid \alpha$, interleukin $\mid \alpha$; DSW, Dead Sea mineral-rich water; PS, PolluStop; NC, negative control; PC, positive control.

both IL-1 $\alpha$ and PGE2 after ozone exposure by $32 \%$ and $24 \%$, respectively (Figures 4 and 5), while treatment with a combination containing DSW $0.25 \%+$ PS 5\% was observed to reduce only PGE2 release by $26 \%$ (Figure 5 ).

\section{Discussion}

The evolution of human lifestyles and the increasing urbanization rate during the last few decades have resulted in pollution becoming a dominant stressor and a cause of concern for our health. Nevertheless, since modern pollution is not specifically defined as one stressor but a complex "cocktail" of many environmental factors, treating pollution health risks is much more challenging. Powerful experimental models

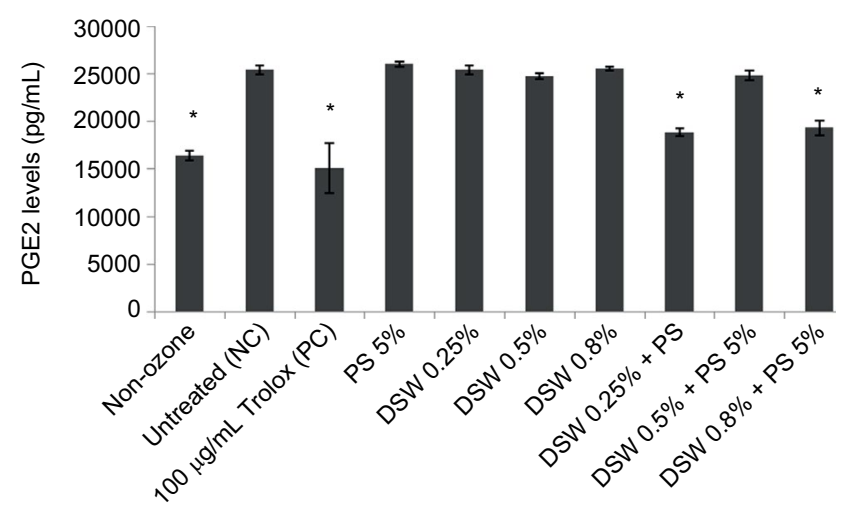

Figure 5 PGE2 levels following ozone exposure to DSW and PS treatments. Notes: Epidermis equivalents were treated topically with test materials. Following overnight incubation, they were exposed to $20 \mathrm{ppm}$ ozone for I hour and test materials were reapplied. After 24 hours of incubation, the medium was collected for PGE2 analysis. PGE2 values are expressed as concentrations. Values are expressed as mean \pm standard deviation. *Values that are significantly different from the untreated tissues $(p<0.05)$.

Abbreviations: PGE2, prostaglandin $E_{2}$; DSW, Dead Sea mineral-rich water; PS, PolluStop; NC, negative control; PC, positive control.

are needed to elucidate the damage mechanisms and plan an effective strategy to protect human skin from contemporary urban pollution damages. Skin as the outermost barrier is in direct contact with various air pollutants, both primary (e.g., PM, toxic gases such as nitrogen oxides) and secondary (e.g., ozone, PANs). In order to develop an effective treatment against pollution for skin, the damage mechanisms have to be better understood. Therefore, we chose to analyze skin response to various pollution factors using different skin models. Most existing data in the scientific literature are based on clinical studies ${ }^{6,9-11}$; however, studies dealing with alternative laboratory skin models are scarce. There is a need for cost-effective and high-throughput tools for the elucidation of relevant biomarkers induced by different polluting factors. Thus, the importance of valid skin laboratory models for studying the risks of urban pollution is more significant than ever. Laboratory skin pollution models can also be used to evaluate treatment regimens and to screen large amounts of topical preparations before proceeding to the expensive stage of clinical trials. So far there is no validated model for pollution in skin. We recently attempted to establish a pollution skin model treated with a standard cigarette smoke solution, in our skin laboratory, using ex vivo human skin organ culture. However, the significant damage biomarkers are not yet fully characterized (unpublished data). In this study, a well-established commercialized in vitro reconstructed epidermis was used as a representative skin model for two common pollution types:

1. MOP containing heavy metals and atmospheric PM, for primary pollution and

2. ozone exposure, for secondary pollution. 
These two different skin models are complementary and thus might provide more valuable data regarding the selectivity of antipollution protection mode of action by different test materials. The in vitro test results may supply a valuable source for prediction of antipollution protection manner in future cosmetic and therapeutic applications.

Oxidation and inflammation biomarkers are reported to be induced following exposure to various pollutants. ${ }^{22-24}$ Detection of such biomarkers can serve as an important indication for the assessment of the damage caused by different pollution types and the antipollution protective effect of potent substances. IL- $1 \alpha$ and PGE2 were evaluated in this study as representative biomarkers for inflammation induced by pollution.

Epidermis exposure to an MOP (PM and heavy metals) induced significantly the release of IL- $1 \alpha$, together with a decrease in cell viability. Other studies also exhibited the elevation in IL- $1 \alpha$ release as a result of exposure to PM. This can be due to reactive oxygen species (ROS) overproduction that leads to activation of transcription factors such as nuclear factor-kappa B (NF- $\mathrm{KB}$ ) and activator protein 1 (AP-1). As a result of translocation of the activated transcription factors, proinflammatory cytokines are synthesized, especially tumor necrosis factor alpha (TNF $\alpha)$, IL- $1 \alpha$, interleukin 6 (IL-6) and interleukin 8 (IL-8), which are closely related to inflammatory skin diseases, skin aging and skin cancers. ${ }^{25}$ Yang et $\mathrm{al}^{26}$ showed that IL- $1 \alpha$ levels were higher in smokers' skin comedones. Campbell et al ${ }^{27}$ showed that IL- $1 \alpha$ levels together with NF-kB levels were increased in the brain tissue of mice exposed to airborne PM compared to those of controlled mice, suggesting that PM triggers a proinflammatory response in the nervous tissue via the involvement of IL- $1 \alpha$.

Ozone exposure of the reconstructed epidermis tissues resulted in a significant increase in the release of the inflammatory markers IL-1 $\alpha$ and PGE2, along with a significant decrease in tissue viability. This is in line with other studies: Manzer et $\mathrm{a}^{28}$ showed that ozone had stimulated the secretion of IL-1 $\alpha$ in rat alveolar macrophage cell line. Additionally, the effect of acute ozone exposure on the arachidonic acid cascade was shown on airway segments of rats where chronic ozone exposure enhanced the release of PGE2. ${ }^{29}$

Here, two commercial materials were tested for antipollution protection: DSW (Dead Sea water extract) and PS (biosaccharide gum-4 or 1,2-hexanediol). Dead Sea ingredients are known for their therapeutic and beneficial cosmetic effects. PS is known for protective properties against specific pollution stress, i.e., atmospheric PM and heavy metals, as demonstrated by the manufacturer (unpublished data). Additionally, DSW in different concentration was combined with PS.

DSW alone has a protective effect against skin exposure to a mixture of heavy metals and atmospheric PM, which are known as inducing skin pollution stress. The protection was by inhibition of IL- $1 \alpha$ release (Figure 2). Similarly, as expected, the composition comprising the specific polysaccharide of PS illustrated a protective effect against exposure to a mixture of heavy metals and atmospheric PM on reconstructed epidermis. This branched out polymer is believed to provide a mechanical defense (like a "protective armor"), preventing the contact/penetration of PM and heavy metals with the epidermis. This protection activity was retained with compositions, which comprised combinations of the DSW and PS.

Remarkably, the skin-protecting capability of DSW and PS, as a stand-alone treatment, was completely different in the other model of secondary pollution by ozone exposure: neither DSW nor PS exhibited any protection against the inflammatory markers IL-1 $\alpha$ (Figure 4) and PGE2 (Figure 5). Interestingly, a combination of DSW and PS demonstrates significant protection from ozone exposure according to both markers. Further bewildering is the absence of a clear trend of the protection capacity vs concentrations. A total of $0.5 \%$ DSW and $5 \%$ PS show superior protection to those of $0.25 \%$ DSW $+5 \%$ PS and $0.8 \%$ DSW $+5 \%$ PS, which have a comparable effect on PGE2 levels. This phenomenon illustrates the complexity of understanding biological activity of a formulation containing numerous active ingredients. In order to further elucidate the activity of DSW and PS together, additional experiments are necessary that include tracking more biomarkers (immunomodulators) and various DSW/PS concentration ratios. The most effective protection against ozone exposure, measured by attenuating the induction of both IL-1 $\alpha$ and PGE2, was achieved using a combination of DSW $0.8 \%$ and PS 5\%. As seen in Figures 4 and 5, the extent of the protective effect is similar to that measured with Trolox, which serves as a standard reference for antioxidant protection.

From the observations, it can be concluded that the combination of DSW $0.8 \%+$ PS $5 \%$ is the only compound that protected from all types of pollutants tested in this study: MOP and ozone. This combination inhibited all tested inflammatory markers: IL- $1 \alpha$ induction by MOP and IL- $1 \alpha$ and PGE2 induction by ozone. The other combinations provided only partial protection against the tested pollutants. DSW $0.25 \%+$ PS $5 \%$ inhibited only IL- $1 \alpha$ induced by MOP and PGE2 induced by ozone but not IL-1 $\alpha$ induced by ozone. 
DSW $0.5 \%+$ PS 5\% inhibited only IL- $1 \alpha$ induced by MOP but not IL-1 $\alpha$ and PGE2 induced by ozone.

\section{Conclusion}

A selective strategy for skin protection against various pollution health risks was demonstrated in this work as a tool to cope with the complexity of modern pollution protection needs. Using two different test materials on two different topical pollution skin models revealed a significant protection capability of each material against primary pollution as stand-alone, while only their combination was effective against the exposure to secondary pollution. This leads to the conclusion that many active materials cannot solely provide a broad protection against different types of pollutants. However, by combining these active materials and adjusting their ratios, anti-pollution activity is optimized and can battle more pollutants. This observation sheds a new light on antipollution strategy relying on selectivity. Because contemporary human skin is constantly exposed to dynamic pollution, which varies during daytime, local zone, season, etc., using materials providing selective protection against different pollution types and components might be beneficial for future antipollution regimen formulated in both pharmaceutical and cosmetic products.

\section{Acknowledgment}

The authors thank Mr Robert Holtz of BioInnovation Laboratories Inc., Lakewood, USA; Dr Paola Perelli Cippo and Dr Elena Allifranchini of Abich Biological and Chemical Analysis, Verbania, Italy and Solabia Group, Pantin Cedex, France.

\section{Disclosure}

The authors report no conflicts of interest in this work.

\section{References}

1. Krutmann J, Liu W, Li L, et al. Pollution and skin: from epidemiological and mechanistic studies to clinical implications. J Dermatol Sci. 2014;76(3):163-168.

2. WHO. Burden of Disease from Household Air Pollution for 2012. 2014. Available from: http://www.who.int/phe/health_topics/outdoorair/databases/ FINAL_HAP_AAP_BoD_24March2014.pdf. Accessed May 9, 2017.

3. Jux B, Kadow S, Luecke S, Rannug A, Krutmann J, Esser C. The aryl hydrocarbon receptor mediates UVB radiation-induced skin tanning. J Invest Dermatol. 2011;131(1):203-210.

4. Nie H, Fu S, Dong Y, Yang Z. Polychlorinated biphenyls in respirable particulate matter from different industrial areas in northern China. Chemosphere. 2014;114:210-218.

5. Moller P, Loft S. Oxidative damage to DNA and lipids as biomarkers of exposure to air pollution. Environ Health Perspect. 2010;118(8): $1126-1136$.

6. Kramer U, Sugiri D, Ranft U, et al. Eczema, respiratory allergies, and traffic-related air pollution in birth cohorts from small-town areas. J Dermatol Sci. 2009;56(2):99-105.
7. Larrieu S, Lefranc A, Gault G, et al. Are the short-term effects of air pollution restricted to cardiorespiratory diseases? Am J Epidemiol. 2009;169(10):1201-1208.

8. Lu X, Zhang X, Li LY, Chen H. Assessment of metals pollution and health risk in dust from nursery schools in Xi'an, China. Environ Res. 2014;128:27-34.

9. Vierkotter A, Schikowski T, Ranft U, et al. Airborne particle exposure and extrinsic skin aging. J Invest Dermatol. 2010;130(12):2719-2726.

10. Kim J, Kim EH, Oh I, et al. Symptoms of atopic dermatitis are influenced by outdoor air pollution. J Allergy Clin Immunol. 2013;132(2): 495-8.e1.

11. Xu F, Yan S, Wu M, et al. Ambient ozone pollution as a risk factor for skin disorders. Br J Dermatol. 2011;165(1):224-225.

12. Valacchi G, van der Vliet A, Schock BC, et al. Ozone exposure activates oxidative stress responses in murine skin. Toxicology. 2002;179(1-2):163-170.

13. Halevy S, Sukenik S. Different modalities of spa therapy for skin diseases at the Dead Sea area. Arch Dermatol. 1998;134(11):1416-1420.

14. Ma'or Z, Yehuda S, Voss W. Skin smoothing effects of Dead Sea minerals: comparative profilometric evaluation of skin surface. Int J Cosmet Sci. 1997;19(3):105-110.

15. Moses SW, David M, Goldhammer E, Tal A, Sukenik S. The Dead Sea, a unique natural health resort. Isr Med Assoc J. 2006;8(7):483-488.

16. Sukenik S, Giryes H, Halevy S, Neumann L, Flusser D, Buskila D. Treatment of psoriatic arthritis at the Dead Sea. J Rheumatol. 1994;21(7):1305-1309.

17. Soroka Y, Ma'or Z, Leshem Y, et al. Aged keratinocyte phenotyping: morphology, biochemical markers and effects of Dead Sea minerals. Exp Gerontol. 2008;43(10):947-957.

18. Sutherland IW, inventor; National Research Development Corp., assignee. Gel-forming polysaccharides. United States patent US 4638059 A. 1987 Jan 20.

19. Schmitt A, Hauser C, Jaunin F, Dayer JM, Saurat JH. Normal epidermis contains high amounts of natural tissue IL 1 biochemical analysis by HPLC identifies a MW approximately $17 \mathrm{Kd}$ form with a P1 5.7 and a MW approximately $30 \mathrm{Kd}$ form. Lymphokine Res. 1986;5(2):105-118.

20. Flower RJ, Harvey EA, Kingston WP. Inflammatory effects of prostaglandin D2 in rat and human skin. Br J Pharmacol. 1976;56(2):229-233.

21. Honda T, Matsuoka T, Ueta M, Kabashima K, Miyachi Y, Narumiya S. Prostaglandin E(2)-EP(3) signaling suppresses skin inflammation in murine contact hypersensitivity. J Allergy Clin Immunol. 2009;124(4): 809-18.e2.

22. Becker S, Dailey LA, Soukup JM, Grambow SC, Devlin RB, Huang YC. Seasonal variations in air pollution particle-induced inflammatory mediator release and oxidative stress. Environ Health Perspect. 2005;113(8):1032-1038.

23. Chuang KJ, Chan CC, Su TC, Lee CT, Tang CS. The effect of urban air pollution on inflammation, oxidative stress, coagulation, and autonomic dysfunction in young adults. Am J Respir Crit Care Med. 2007;176(4):370-376.

24. Risom L, Moller P, Loft S. Oxidative stress-induced DNA damage by particulate air pollution. Mutat Res. 2005;592(1-2):119-137.

25. Kim KE, Cho D, Park HJ. Air pollution and skin diseases: adverse effects of airborne particulate matter on various skin diseases. Life Sci. 2016;152:126-134.

26. Yang YS, Lim HK, Hong KK, et al. Cigarette smoke-induced interleukin-1 alpha may be involved in the pathogenesis of adult acne. Ann Dermatol. 2014;26(1):11-16.

27. Campbell A, Oldham M, Becaria A, et al. Particulate matter in polluted air may increase biomarkers of inflammation in mouse brain. Neurotoxicology. 2005;26(1):133-140.

28. Manzer R, Dinarello CA, McConville G, Mason RJ. Ozone exposure of macrophages induces an alveolar epithelial chemokine response through IL-1alpha. Am J Respir Cell Mol Biol. 2008;38(3):318-323.

29. Szarek JL, Valentovic MA. Release of prostaglandin E2 and leukotriene C4/D4 from airway segments isolated from rats after exposure to ozone for 20 months. Toxicology. 1995;100(1-3):111-119. 


\section{Publish your work in this journal}

Clinical, Cosmetic and Investigational Dermatology is an international, peer-reviewed, open access, online journal that focuses on the latest clinical and experimental research in all aspects of skin disease and cosmetic interventions. This journal is included on PubMed. The manuscript management system is completely online and includes a very quick and fair peer-review system, which is all easy to use. Visit http://www.dovepress.com/testimonials.php to read real quotes from published authors

Submit your manuscript here: https://www.dovepress.com/clinical-cosmetic-and-investigational-dermatology-journal 